\title{
Theory of Molecular Scattering from and Photochemistry at Ice Surfaces
}

\author{
G. J. Kroes ${ }^{1}$ and S. Andersson ${ }^{1,2}$ \\ ${ }^{1}$ Leiden Institute of Chemistry, Gorlaeus Laboratories, Leiden University, \\ P.O. Box 9502, 2300 RA Leiden, The Netherlands \\ email: g.j.kroes@chem.leidenuniv.nl \\ ${ }^{2}$ Leiden Observatory, Leiden University, P.O. Box 9513, 2300 RA Leiden, The Netherlands
}

\begin{abstract}
The classical trajectory methodology for studying scattering of ions, atoms, or molecules from ice surfaces, and photodissociation of water at or in the surface of ice, is presented. The forces between the collider and the water molecules, or between the fragments of a dissociating molecule and the surrounding water molecules, are based on pair potentials taken either from $a b$ initio calculations or derived empirically. Dynamical observables like sticking probabilities and kinetic energy distributions of desorbing photo-fragments are computed by solving Newton's equations of motion, starting from representative initial conditions. Four studies with relevance to astrochemistry are considered: the sticking of $\mathrm{H}$ atoms to ice $\mathrm{Ih}$, the sticking of $\mathrm{CO}$ to ice $\mathrm{Ih}$ and ice $\mathrm{I}_{a}$, the sticking of protons to ice $\mathrm{Ih}$, and the photodissociation of water in ice $\mathrm{Ih}$ and ice $\mathrm{I}_{a}$, also with a view to the subsequent chemistry of the $\mathrm{H}$ - and $\mathrm{OH}$-products with co-adsorbed molecules. Where possible, the theoretical results are compared with experiments.
\end{abstract}

Keywords. astrochemistry — ISM: molecules — methods: numerical — molecular processes

\section{Introduction}

The formation of molecules in the interstellar medium (ISM) can proceed through several classes of reactions (van Dishoeck 1998). An important class consists of surface reactions, which take place on dust particles. In dense clouds, these dust particles, can be covered by icy mantles (Ehrenfreund \& Schutte 2000). The ice can consist of $\mathrm{H}_{2} \mathrm{O}$ molecules, but molecules like $\mathrm{CO}, \mathrm{CO}_{2}, \mathrm{NH}_{3}$, and $\mathrm{CH}_{4}$ may also be present. The icy mantles are generally believed to have an amorphous structure (Hagen et al. 1981). However, crystalline ice (ice Ih) can also be relevant to astrochemistry. For instance, amorphous ice (ice $\mathrm{I}_{a}$ ) deposited on amorphous silicate particles can be phase transformed to crystalline ice at temperatures close to $110 \mathrm{~K}$ (Maldoni et al. 1999). On dust particles in the ISM, this temperature can be achieved through the impact of cosmic rays. In this paper, we will therefore consider processes occurring on both amorphous and crystalline pure water ice.

An important example of a surface reaction taking place on dust particles is the formation of molecular hydrogen, the most abundant molecule in the ISM. It is generally assumed that the $\mathrm{H}_{2}$ in the ISM is formed through surface reactions occurring on grains (Hollenbach \& Salpeter 1971; Herbst 1995). Because the mechanism of hydrogen recombination always involves the trapping of at least one hydrogen atom at the surface prior to reaction, the study of trapping (sticking) of $\mathrm{H}$ to ice is relevant to the astrochemistry of dense clouds. We will present results (Al-Halabi et al. 2002) of calculations on the trapping of $\mathrm{H}$ on ice $\mathrm{Ih}$, and compare these with calculations as well as experiments on the trapping of $\mathrm{H}$ on ice $\mathrm{I}_{a}$. 
Solid CO has been observed along many lines of sight towards the dense regions in the ISM, the Galactic center, and high- and low-mass young stellar objects (Tanaka et al. 1994; Pontoppidan et al. 2003). Observations suggest that $\mathrm{CO}$ resides in two distinct environments; i.e., in van der Waals bonded ice (also called apolar ice or $\mathrm{H}_{2} \mathrm{O}$-poor ice), and in hydrogen bonded ice (also called polar ice or $\mathrm{H}_{2} \mathrm{O}$-rich ice). One question concerning the hydrogen bonded ice is related to the mixing of $\mathrm{CO}$ and $\mathrm{H}_{2} \mathrm{O}$ : does an intimate mixture form, or is $\mathrm{CO}$ present in multilayer form on the surface of $\mathrm{H}_{2} \mathrm{O}$ ice? Clever experiments using coadsorbates which either bond or do not bond to surface dangling OH-groups of $\mathrm{H}_{2} \mathrm{O}$ ice (Devlin 1992; Graham et al. 1999) strongly suggest that intimately mixed ice should exhibit a $2152 \mathrm{~cm}^{-1}$ feature in the infrared due to the interaction of $\mathrm{CO}$ with dangling $\mathrm{OH}$ bonds, while a $2139 \mathrm{~cm}^{-1}$ feature should be due to $\mathrm{CO}-\mathrm{CO}$ interactions in solid $\mathrm{CO}$ ice, or interactions of $\mathrm{CO}$ with "bonded $\mathrm{OH}^{\circ}$. These experiments also suggest that the dangling $\mathrm{OH}$-bonds should be the preferred adsorption sites of isolated CO-molecules adsorbed to $\mathrm{H}_{2} \mathrm{O}$ ice. Molecular dynamics (MD) calculations employing accurate pair potentials can provide support for the above interpretation of laboratory experiments and observations, and yield insight into the dynamics of trapping of $\mathrm{CO}$ on ice. Such calculations (Al-Halabi et al. 2003; Al-Halabi, van Dishoeck, \& Kroes 2004; Al-Halabi et al. 2004) are also reported here.

Interactions of protons with water ice are also relevant to astrochemistry. Cosmic rays (CRs) consist predominantly of high-energy protons. The CR-ice interaction is the predominant agent leading to mass loss of water-ice grains (Mukai \& Schwehm 1981). Impact of $\mathrm{CRs}$ on mixed ices can promote the formation of molecules like carbonic acid $\left(\mathrm{H}_{2} \mathrm{CO}_{3}\right)$ (Gerakines et al. 2000) and amino acids (Kobayashi et al. 1995). We will therefore also present new, surprising results for the sticking of $\mathrm{H}^{+}$ions to ice $\mathrm{Ih}$ (Cabrera Sanfelix et al. 2005). The results are for a range of energies $(0.05-4.0 \mathrm{eV})$ which is much lower than the kinetic energy of CR protons. The results are nevertheless of interest. First, they show that a spin-off of research on systems of astrochemical interest may be that new discoveries in chemical physics are made, although some of these discoveries might apply to conditions that are not of direct interest to astrochemistry. Second, we expect that many of the conclusions derived from our study (Cabrera Sanfelix et al. 2005) should also be applicable to low-energy collisions of $\mathrm{H}^{+}$-containing molecular ions (such as $\mathrm{H}_{3}{ }^{+}$) with ice.

Photodissociation of water in ice, which can be induced by UV light of energy exceeding $7.6 \mathrm{eV}$ (Kobayashi 1983), is also of interest to astrochemistry. The products of the reaction ( $\mathrm{H}$ and $\mathrm{OH}$, and possibly other products as well) can go on to react with co-adsorbed or co-absorbed molecules. It has been postulated that $\mathrm{CO}_{2}$ could be formed in this way, i.e., by reaction of $\mathrm{OH}$ with $\mathrm{CO}$ present on or in $\mathrm{H}_{2} \mathrm{O}$ rich ice (Allamandola et al. 1988), and experiments on UV irradiation of mixed $\mathrm{CO}: \mathrm{H}_{2} \mathrm{O}$ ice have indeed shown efficient $\mathrm{CO}_{2}$ formation (Watanabe \& Kouchi 2002). We here present results on the dynamics of photodissociation of $\mathrm{H}_{2} \mathrm{O}$ in ice, with much attention paid to the dynamics of the $\mathrm{H}$ and $\mathrm{OH}$ fragments, also with a view to their possible subsequent chemistry with co-adsorbates (Andersson et al. 2005; Andersson et al., in prep.) The calculations to be presented may be viewed as a first step in the modeling of photoinitiated reactions of products of water photolysis with co-adsorbed molecules.

We first discuss the molecular dynamics (MD) method we use in our calculations on sticking and photodissociation of $\mathrm{H}_{2} \mathrm{O}$ in ice, in $\S 2$. Results of our MD calculations are presented in $\S 3$, for $\mathrm{H}+$ ice $\mathrm{Ih}(\S 3.1), \mathrm{CO}+$ ice $\mathrm{Ih}$ and ice $\mathrm{I}_{a}(\S 3.2), \mathrm{H}^{+}+$ice $\mathrm{Ih}(\S 3.3)$, and photodissociation of water in ice $\mathrm{Ih}$ and $\mathrm{I}_{a}(3.4)$. We conclude with a summary $(\S 4)$. 


\section{Method}

In this section, we discuss how an MD simulation of molecular processes on or in ice is performed. Whichever problem is studied, such simulations always share common features. First of all, the initial state of the system is prepared, according to the problem of interest. This includes the setting up of a dynamic ice surface ( $\S 2.1)$, and the choice of other initial conditions, such as the selection of the coordinates and momenta of the collider (for calculations on sticking) (§ 2.4). Subsequently, Newton's equations of motion have to be solved for a long enough period of time, and relevant observables have to be extracted ( $(2.2)$. This can only be done if the forces between the molecules are defined, which we do through pair potentials (§ 2.3).

\subsection{The Ice Surface}

The crystalline and amorphous ice surfaces were modeled using the MD method (Allen \& Tildesley 1987). Ice Ih is always modeled as proton disordered (see Kroes 1992). The lower part of the ice Ih surface is usually modeled by 2 bilayers of $\mathrm{H}_{2} \mathrm{O}$ molecules (120 molecules) which are held fixed. The upper part of the surface is modeled by 4 or 6 bilayers of $\mathrm{H}_{2} \mathrm{O}$ molecules (240 or 360 molecules) which are allowed to move according to Newton's equations of motion. The $\mathrm{H}_{2} \mathrm{O}$ molecules are modeled as rigid rotors, with only one exception: if the photodissociation of water in ice is modeled, the photoexcited molecule's intramolecular motion following photoexcitation is also modeled in order to follow the dissociation dynamics and to enable the computation of the absorption spectrum. The $\mathrm{H}_{2} \mathrm{O}$ molecules are put in a box which is replicated in two directions according to periodic boundary conditions, to create an infinite ice surface. The TIP4P pair potential (Jorgensen et al. 1983) is used to model the forces between the water molecules, because its use yields stable ice Ih at the relevant temperatures (Kroes 1992; Karim \& Haymet 1988). For ice Ih, the initial ice configuration obeys the famous ice rules (Bernal \& Fowler 1933), and has a zero dipole moment (Kroes 1992). The surface temperature $\left(T_{s}\right)$ of interest is imposed on the ice surface through the use of the computational analogue of a thermostat (Berendsen et al. 1984). For details and for a more technical discussion of the setting up of a surface of ice Ih, the reader is referred to Kroes (1992).

In Figure 1, top $(1 a)$ and side views $(1 c)$ of ice Ih are shown, where it is the (0001) basal plane face of ice that is exposed to the vacuum. Ice Ih has a bilayer structure, the bilayers being about $3.5 \AA$ thick. Another important observation is that the water molecules in the upper monolayer can either have one $\mathrm{H}$ atom pointing away from the surface (dangling $\mathrm{OH}$ ), or both $\mathrm{H}$ atoms pointing obliquely down to the surface. This has important consequences for adsorption of $\mathrm{CO}$ and protons (see $\S 3$ ).
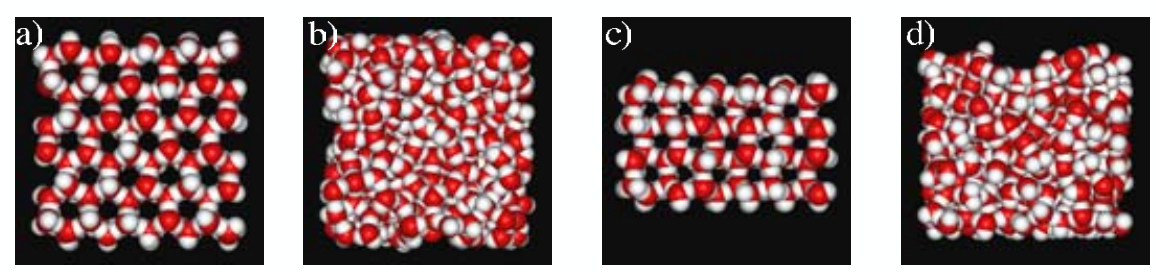

Figure 1. Top views $(a$ and $b)$ and side views $(c$ and $d)$ are shown of simulated ice Ih $(a$ and $c$ ) and ice $\mathrm{I}_{a}(b$ and $d)$, at $T_{s}=90 \mathrm{~K}$. In the figures, the dark atoms are the oxygen atoms, and the light atoms the hydrogen atoms.

Interstellar $\mathrm{H}_{2} \mathrm{O}$ ice is believed to be predominantly amorphous in structure (Hagen et al. 1981). The specific morphology of amorphous ice remains an open 
question. The $\mathrm{H}_{2} \mathrm{O}$ ice is thought to form through chemical reactions of $\mathrm{H}$ and $\mathrm{O}$ atoms on dust grains, but it is not known to exactly which structure this would lead. One usually assumes that the structure closely resembles that of ice grown by vapor deposition in the laboratory, known as amorphous solid water (ASW), rather than ice phases formed under high-pressure condistions, such as high-density amorphous (hda) ice, or low-density amorphous (lda) ice (Petrenko \& Whitworth 1999; Ehrenfreund et al. 2003). We make amorphous ice (Al-Halabi, van Dishoeck, \& Kroes 2004; Al-Halabi et al. 2004) by first heating hexagonal ice to room temperature, resulting in melting, and subsequent fast cooling (hyperquenching) to the desired temperature, using the computational analogue of a thermostat (Berendsen et al. 1984). The calculated average density of our amorphous ice is about $0.93 \mathrm{~g} / \mathrm{cm}^{3}$, which is in the range of measured densities $\left(0.85-1.05 \mathrm{~g} / \mathrm{cm}^{3}\right)$ of dense amorphous solid water deposited at $T=22 \mathrm{~K}$ for a wide range of deposition angles (Dohnálek et al. 2003). Just as for the case of ice Ih, the surface consists of a box of molecules which is replicated in two directions.

In Figure 1, top $(1 b)$ and side views $(1 d)$ of the dense ice $\mathrm{I}_{a}$ we made are shown. The amorphous ice completely lacks the regularity seen in ice Ih. The surface of ice $\mathrm{I}_{a}$ is irregular, displaying holes, into which $\mathrm{CO}$ can move and interact with many $\mathrm{H}_{2} \mathrm{O}$ molecules simultaneously. Most of the water molecules hydrogen bond to 4 neighbouring water molecules, while a substantial fraction hydrogen bond to only 3 neighbouring water molecules (Al-Halabi et al. 2004).

\subsection{Classical Dynamics and Calculation of Absorption Spectrum}

To simulate the dynamics of a collision or photodissociation event, Newton's equations of motion are integrated in time. The integration is started from coordinates and momenta that are representative of the initial conditions (§ 2.4). Sticking probabilities and dynamical quantities related to photodissociation are determined by following the trajectory in time. For instance, to determine a sticking probability $\left(P_{s}\right)$, first an operational definition is made of sticking. A requirement for sticking to occur in the MD is that a trajectory exhibit multiple turning points in the curve showing the molecule-surface distance as a function of time. An additional criterion may be that the final energy of the adsorbed molecule is less than a predefined threshold value. For the actual definition adopted in individual cases, the reader is referred to Al-Halabi et al. (2002), Al-Halabi et al. (2003), Al-Halabi, van Dishoeck, \& Kroes (2004), Al-Halabi et al. (2004), and Cabrera Sanfelix et al. (2005). The calculation of dynamical quantities associated with photodissociation of $\mathrm{H}_{2} \mathrm{O}$ in ice is discussed in Andersson et al. (2005) and Andersson et al., in prep.

The absorption spectrum is calculated according to Schinke (1993):

$$
\sigma_{\text {tot }}^{(i)}(\omega) \propto E_{\text {photon }} \int d \tau P_{W}^{i}(\tau) \mu_{i f}^{2} \delta\left[H_{f}(\tau)-E_{f}\right]
$$

Here, $P_{W}^{i}(\tau)$ is the probability distribution function associated with the initial $(i)$ vibrational state of the molecule in its ground electronic state, and $H_{f}(\tau)$ the Hamilton function in the upper electronic state $(f)$. Furthermore, $\mu_{i f}$ is the transition dipole function, $E_{i}$ the energy of the initial state, and $E_{f}=E_{i}+E_{\text {photon }} . P_{W}^{i}(\tau)$ is calculated from a Wigner (semi-classical) distribution function fitted to the ground state vibrational wave function of the water molecule to be photodissociated, as discussed in van Harrevelt et al. (2001). The integration is over the entire phase space. The transition dipole moment function was taken from van Harrevelt \& van Hemert (2000). The spectrum is calculated by determining the energy difference between the excited state potential and the ground state potential for a large number of phase space points, and the absorption spectrum is calculated as the number of times excitation energies occur within an energy 
Table 1. Essential features of the pair potentials for the interaction of the collider X with an individual water molecule. Below, $R_{\min }$ refers to the distance between a defined atom pair, in the potential minimum geometry.

\begin{tabular}{|c|c|c|c|c|c|}
\hline $\mathrm{X}$ & $\begin{array}{c}\text { Method of } \\
\text { derivation }\end{array}$ & $\begin{array}{c}\text { Potential minimum } \\
\left(\mathrm{kJ} \mathrm{mol}^{-1}\right)\end{array}$ & $\begin{array}{c}R_{\min } \\
(\AA)\end{array}$ & $\begin{array}{c}\text { Atom pair } \\
R_{\text {min }} \\
\text { refers to }\end{array}$ & References \\
\hline $\mathrm{CO} \mid \mathrm{HF}+\mathrm{MP2}$ & -5.1 & 2.47 & $\mathrm{C}-\mathrm{H}_{w}$ & Al-Halabi et al. $(2003)$ \\
\hline $\mathrm{H}$ & $\mathrm{UHF}+\mathrm{MP} 4$ & -0.63 & 3.4 & $\mathrm{H}^{-} \mathrm{O}_{w}$ & $\begin{array}{c}\text { Al-Halabi et al. }(2002) \\
\text { Zhang et al. } 1991\end{array}$ \\
\hline $\mathrm{H}^{+}$ & empirical & -705 & 1.04 & $\mathrm{H}^{+}-\mathrm{O}_{w}$ & $\begin{array}{c}\text { Cabrera Sanfelix et al. }(2005) \\
\text { Kozack \& Jordan (1992) }\end{array}$ \\
\hline
\end{tabular}

interval of a predetermined width. The approach outlined above has been shown to give good agreement between classical and quantum treatments of gas phase photodissociation (Schinke 1993). For more details on how we apply this procedure, see also Andersson et al. (2005) and Andersson et al., in prep.

\subsection{Pair Potentials}

For the integration of the equations of motion, it is necessary to specify the forces that act between the water molecules belonging to the ice, and between the water molecules and the collider (sticking) or between the water molecules and the water molecule that was (or is to be) excited. The potential from which these forces are derived is always based on pair potentials acting between the collider (or the water molecule singled out for photoexcitation) and an $\mathrm{H}_{2} \mathrm{O}$ molecule. The total interaction of the collider (or the special water molecule) with the ice surface is then taken as a sum over all (other) $\mathrm{H}_{2} \mathrm{O}$ molecules.

The pair potentials used in the calculations on sticking are either taken from $a b$ initio calculations or derived empirically. The validity of the pair potential is usually checked by comparison to experimental results. For instance, the $\mathrm{CO}-\mathrm{H}_{2} \mathrm{O}$ pair potential that was used (Al-Halabi et al. 2003; Al-Halabi, van Dishoeck, \& Kroes 2004; Al-Halabi et al. 2004) was based on ab initio HF + MP2 calculations, and the minimum geometry obtained from the pair potential was checked to compare favorably with Fourier transform microwave absorption spectroscopy experiments (Yaron et al. 1990). Details on the pair potentials used in the calculations for $\mathrm{H}, \mathrm{CO}$, and $\mathrm{H}^{+}+\mathrm{H}_{2} \mathrm{O}$ can be found in Al-Halabi et al. (2002), Al-Halabi et al. (2003), and Cabrera Sanfelix et al. (2005), respectively. Some essential features of the pair potentials are summarized in Table 1.

In the calculations on photodissociation of water in ice, the potential energy of the water molecule singled out for photoexcitation consists of an intramolecular and an intermolecular part. In the computation of the photoabsorption spectrum, the intramolecular potentials of the photoexcited $\mathrm{H}_{2} \mathrm{O}$ molecule in its ground electronic and first excited states are taken from the work of Dobbyn \& Knowles (1997).

For the molecule that is photoexcited, the intermolecular interaction with the surrounding water molecules is taken from the TIP3P model (Jorgensen et al. 1983), to avoid the complication, arising with the TIP4P model, that the negative charge is not on the (moving) $\mathrm{O}$ atom. In the calculation of the absorption spectrum and in the MD calculation on the dynamics following photoexcitation, the excited water molecule interacts with the surrounding water molecules as an excited state water molecule would. In the first potential model we used, charges were put on the $\mathrm{O}$ and $\mathrm{H}$ atoms of the 
excited molecule (Andersson et al. 2005) to mimic the dipole moment exhibited by gas phase water in its first excited electronic state (Klein et al. 1996). In our second potential model, smaller charges $(-0.2 \mathrm{e}$ on $\mathrm{O}$ and $0.1 \mathrm{e}$ on $\mathrm{H})$ were used such that the theoretical spectrum was in good agreement with the experimental spectrum (Kobayashi 1983). For a detailed discussion of the pair interaction between excited $\mathrm{H}_{2} \mathrm{O}$ and the surrounding $\mathrm{H}_{2} \mathrm{O}$ molecules, the reader is referred to Andersson et al., in prep.

\subsection{Initial Conditions}

The initial coordinates and momenta from which trajectories are started are selected to represent the physical situation of interest, using a Monte Carlo procedure (Porter \& Raff 1976). The initial conditions are obtained by equilibrating the ice surface in a separate MD run, after a $T_{s}$ has been imposed $(\S 2.1)$. For simulating the collision of an atom, ion, or molecule with the surface, the collider's initial momentum is selected according to the collision energy and incidence angle of interest. If the collider is a molecule, its initial orientation is taken at random from uniform distributions of $\cos \theta$ and $\phi$, where $\theta$ and $\phi$ are the polar angles of orientation of the molecule's bond axis. The molecule's rotational angular momentum $J_{c}$ is taken as $J_{c}=\hbar \sqrt{J(J+1)}$, where $J$ is the molecule's rotational quantum number. The direction of the angular momentum is taken from a random distribution. The collider's impact site on the surface is likewise selected at random.

In the calculations on the photodissociation of water in ice, the initial coordinates and momenta associated with the centre-of-mass motion and rotation of all $\mathrm{H}_{2} \mathrm{O}$ molecules are obtained using a separate MD run. The water molecule to be excited is selected to be one of the water molecules present in a given layer of the simulated ice. Its initial intramolecular coordinates and vibrational momenta are selected at random from the probability distribution obtained from a semi-classical wave function - the Wigner distribution (van Harrevelt et al. 2001). Therefore, the initial phase-space distribution of the dissociating molecule is based on quantum mechanics.

\section{Results and Discussion}

\section{1. $H+I c e$}

The sticking probability $\left(P_{s}\right)$ computed for scattering of atomic hydrogen from ice Ih at normal incidence over a range of collision energies $E_{i}$ is shown for two different $T_{s}$ in Figure 2 (Al-Halabi et al. 2002). The sticking probability could be fitted quite well to an exponential function of $E_{i}$, i.e., $P_{s}=\alpha \exp \left(-E_{i} / \beta\right)$, with $\beta=175 \mathrm{~K}$ (here and in Fig. $2, \mathrm{~K}$ is used as a unit of energy, $\left.1 \mathrm{~K}=8.31 \mathrm{~J} \mathrm{~mol}^{-1}\right)$. The values extracted for $\alpha$ were 1.5 for $T_{s}=10 \mathrm{~K}$ and 0.85 for $T_{s}=70 \mathrm{~K}$ (the fit for $T_{s}=10 \mathrm{~K}$ is not valid at collision energies significantly lower than $100 \mathrm{~K}$ ). The strong dependence of $\alpha$ on $T_{s}$ illustrates the strong dependence of the sticking probability on $T_{s}$ for $\mathrm{H}$. As a result of its weak interaction with the surface, the $\mathrm{H}$ atom is sensitive to the fact that the water molecules are more dynamic and less packed in the warmer surface. As a result, it is more likely that an upward moving water molecule imparts momentum to the impinging $\mathrm{H}$ atom, and that the interaction of $\mathrm{H}$ with the surface is further weakened because it interacts with fewer water molecules, at higher $T_{s}$.

In Figure 2, the results for ice Ih are also compared with results of computational studies for ice $\mathrm{I}_{a}$ that used the same $\mathrm{H}-\mathrm{H}_{2} \mathrm{O}$ potential, so that differences should in principle be due to differences between ice Ih and ice $\mathrm{I}_{a}$. For $T_{s}=10 \mathrm{~K}$, the results for ice Ih fall between the results of ice $\mathrm{I}_{a}$ of Buch \& Zhang (1991) and of Masuda et al. (1998). The discrepancy with the results of Masuda et al. is due to the use of an 

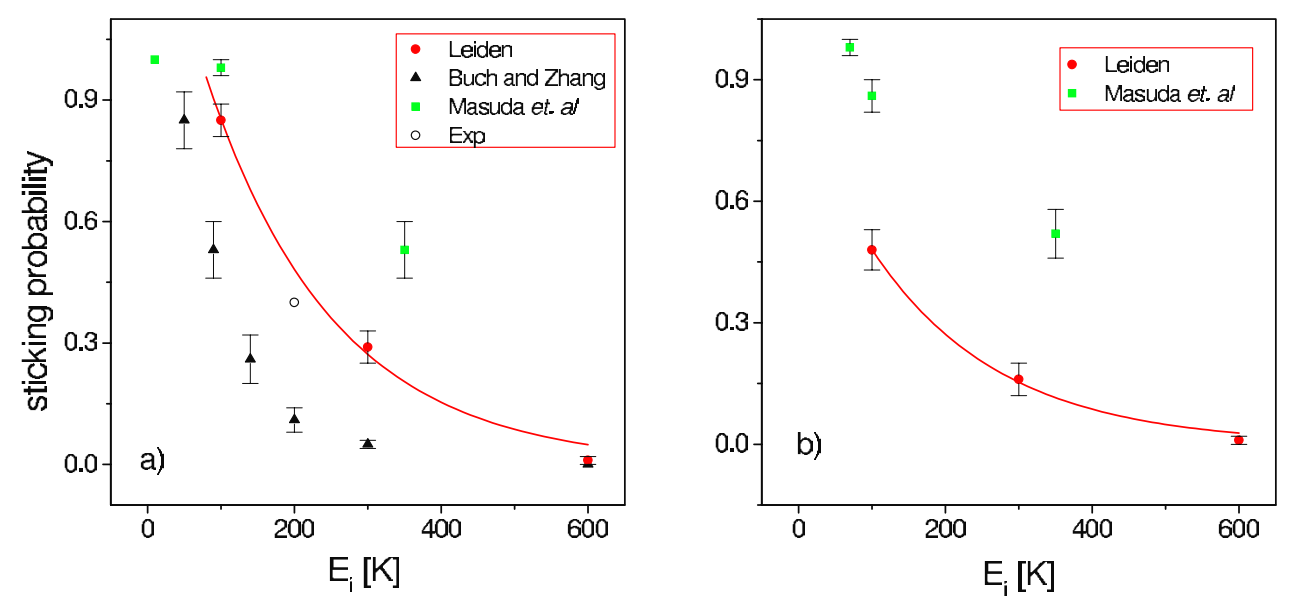

Figure 2. The sticking probability of hydrogen atoms to ice Ih is shown as a function of the collision energy $E_{i}$ for $(a) T_{s}=10 \mathrm{~K}$ and $(b) T_{s}=70 \mathrm{~K}$ (Al-Halabi et al. 2002), together with previous computational results on sticking of hydrogen to ice $\mathrm{I}_{a}$ (Buch \& Zhang 1991; Masuda et al. 1998). A sticking probability extracted by modeling from a TPD experiment using a $200 \mathrm{~K}$ atomic hydrogen beam impinging on an amorphous ice surface at low $T_{s}$ is also shown (Manicó et al. 2001).

erroneous expression of the spherical harmonic, $Y_{00}$, in the equation describing the $\mathrm{H}-$ $\mathrm{H}_{2} \mathrm{O}$ pair potential (Takahashi, private comm.) This expression was printed incorrectly in several papers using the $\mathrm{H}-\mathrm{H}_{2} \mathrm{O}$ interaction potential (Buch \& Zhang 1991; Zhang et al. 1991).

The differences between the sticking probability for ice Ih and ice $\mathrm{I}_{a}$ computed by Buch \& Zhang (1991) is probably due to differences in regularity of the ice surfaces. The amorphous ice cluster studied by Buch \& Zhang is a small cluster consisting of 115 molecules. It was made by successively raining water molecules on to a small nucleus, and the surface of the cluster is quite irregular, with many sites where the impinging $\mathrm{H}$ may experience a significant attractive interaction to only a few water molecules. In contrast, the surface of crystalline ice (Fig. $1 a$ ) is quite regular, so that the impinging $\mathrm{H}$ atom can experience a significant attractive interaction with as many as six $\mathrm{H}_{2} \mathrm{O}$ molecules, if it hits the center of a hexagonal ring. As a result of the stronger interaction, $\mathrm{H}$ exhibits a larger sticking probability on ice Ih than on the ice $\mathrm{I}_{a}$ modeled by Buch \& Zhang. The large difference between trapping of $\mathrm{H}$ on ice $\mathrm{Ih}$ and on ice $\mathrm{I}_{a}$ here observed shows how importantly the ice structure may affect processes that are crucial to astrochemistry: after all, trapping of $\mathrm{H}$ on ice is important to the formation of molecular hydrogen, which is the most abundant molecule in the ISM, in dense clouds.

The difference we found between sticking of $\mathrm{H}$ on ice $\mathrm{Ih}$ and ice $\mathrm{I}_{a}$ also illustrates another important point: it is important to determine how the structure of interstellar amorphous ice is related to the conditions under which it is grown. It is believed that interstellar ice originates from chemical reactions between $\mathrm{H}$ and $\mathrm{O}$ on dust grains; as a result, the surface structure of the ice may be different than obtained by successively raining water molecules onto a pre-existing water nucleus, which could be an appropriate procedure for simulating vapor deposition of $\mathrm{H}_{2} \mathrm{O}$ on ice.

The sticking probability we computed for $T_{s}=10 \mathrm{~K}$ is in good agreement with the sticking probability extracted by modeling thermal desorption experiments on sticking of $\mathrm{H}$ and $\mathrm{D}$ to amorphous ice, using a $200 \mathrm{~K}$ atomic hydrogen beam (Manicó et al. 2001). In the analysis of the experiments, the recombination efficiency $\gamma$ measured was defined 
through $\gamma=P_{s} \eta$, and the assumption was made that the recombination probability $\eta$ can be taken as one, so that $P_{s}=\gamma$. The resulting $P_{s}$ for ice $\mathrm{I}_{a}$ is in good agreement with the theory for ice Ih. Of course, this is not consistent with the difference between the theoretical results for ice Ih and ice $\mathrm{I}_{a}$ as modeled by Buch \& Zhang, i.e., the experimental result for $P_{s}$ on ice $\mathrm{I}_{a}$ significantly differs from the theoretical results of Buch \& Zhang (1991). An open question is whether the agreement between theory and experiment for ice $\mathrm{I}_{a}$ will improve if the ice $\mathrm{I}_{a}$ is set up in a different way.

\section{2. $\mathrm{CO}+\mathrm{Ice}$}

The $P_{s}$ of CO to ice Ih and ice $\mathrm{I}_{a}$ is plotted as a function of $E_{i}$ in Figure 3 , for $T_{s}=90$ K (Al-Halabi, van Dishoeck, \& Kroes 2004). The computed $P_{s}$ can be fitted quite well to an exponential function of $E_{i}$, with $\alpha=1$ and $\beta=90 \mathrm{~kJ} \mathrm{~mol}^{-1}$, respectively, for both ice Ih and ice $\mathrm{I}_{a}$. Interestingly, for $\mathrm{CO}$ the trapping probability appears to be the same for ice $\mathrm{Ih}$ and ice $\mathrm{I}_{a}$. To within the statistical uncertainty implied by the number of classical trajectories run (100 for each energy), the probabilities are the same. In this respect, the trapping of $\mathrm{CO}$ to ice is different from the trapping of $\mathrm{H}$ to ice (Figure $2, \S 3.1$ ). At present, it is unclear whether this difference is due to the difference in the collider, or to the differences in the ice $\mathrm{I}_{a}$ model used in the calculations. In the calculations on $\mathrm{H}$ + ice $\mathrm{I}_{a}$ the ice $\mathrm{I}_{a}$ is modeled as a cluster of $\mathrm{H}_{2} \mathrm{O}$ molecules successively rained on to a pre-existing $\mathrm{H}_{2} \mathrm{O}$ nucleus (Buch \& Zhang 1991), while the ice $\mathrm{I}_{a}$ used in $\mathrm{CO}+$ ice was obtained by rapid quenching of liquid water simulated at room temperature (Al-Halabi, van Dishoeck, \& Kroes 2004).

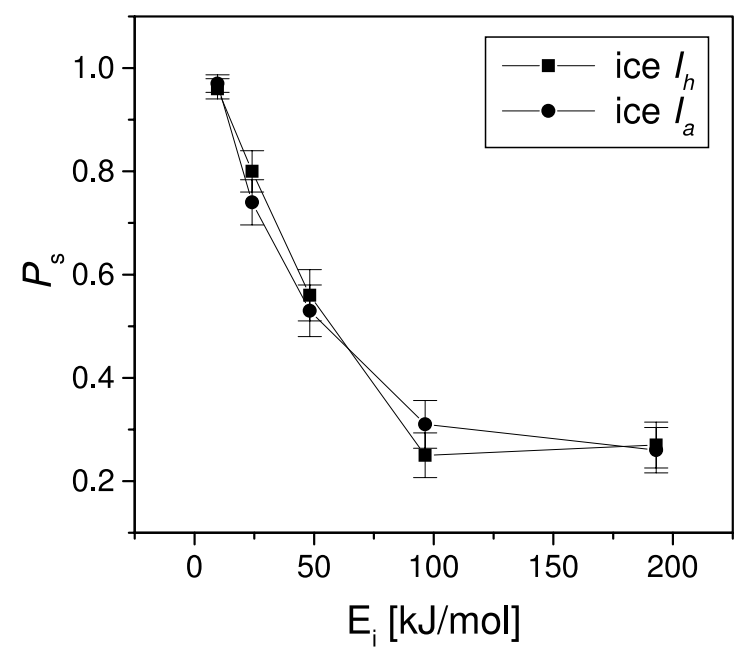

Figure 3. The $P_{s}$ of $\mathrm{CO}$ to ice $\mathrm{Ih}$ and ice $\mathrm{I}_{a}$ is shown as a function of $E_{i}$, for scattering at normal incidence, and $T_{s}=90 \mathrm{~K}$ (Al-Halabi, van Dishoeck, \& Kroes 2004).

For $\mathrm{CO}+$ ice, substantial $P_{s}$ values were obtained for $E_{i}\left(200 \mathrm{~kJ} \mathrm{~mol}^{-1}\right)$, much larger than the average adsorption of $\mathrm{CO}$ to ice, which is about $10 \mathrm{~kJ} \mathrm{~mol}^{-1}$. This is because the energy with which the collider hits the surface can be very rapidly and efficiently dissipated through the hydrogen bonding network of ice, as also found in previous calculations on sticking of $\mathrm{HCl}$ to ice (Al-Halabi et al. 1999; Al-Halabi et al. 2001). In no case was a water molecule found to desorb from the surface, even though the highest $E_{i}$ used was much higher than the energy with which surface water molecules are bound to the bulk of $\mathrm{H}_{2} \mathrm{O}$. An interesting question is whether MD simulations on sticking of atoms and molecules to CO-ice would find a similarly efficient energy dissipation and a similarly stable surface as obtained for $\mathrm{H}_{2} \mathrm{O}$ ice. 
The $P_{s}$ for $\mathrm{CO}$ on ice is smaller than that found for $\mathrm{HCl}+$ ice $(>0.9$ over the entire energy range depicted in Figure 3 (Al-Halabi et al. 1999; Al-Halabi et al. 2001) and effectively larger than that found for $\mathrm{H}+$ ice: the $P_{s}$ of $\mathrm{CO}$ exceeds 0.8 at a value of $E_{i}\left(10 \mathrm{~kJ} \mathrm{~mol}^{-1}\right)$ which is twice as large as the one $\left(600 \mathrm{~K} \approx 5 \mathrm{~kJ} \mathrm{~mol}^{-1}\right)$ where the trapping probability of $\mathrm{H}$ on ice is less than 0.1 (Fig. 2). This order in the efficiency of trapping reflects the interaction strength with the surface, which is correlated to the collider- $\mathrm{H}_{2} \mathrm{O}$ pair interaction (Table 1): the stronger the interaction with the surface, the smaller the part of the sum of the incidence energy and the interaction energy that has to be transferred from incident motion to other motions, for trapping to occur. Therefore, the larger the interaction energy with the surface is, the larger the trapping probability is, for the neutral atoms and molecules we have studied (Al-Halabi et al. 2002; Al-Halabi et al. 2003; Al-Halabi, van Dishoeck, \& Kroes 2004; Al-Halabi et al. 1999; Al-Halabi et al. 2001).
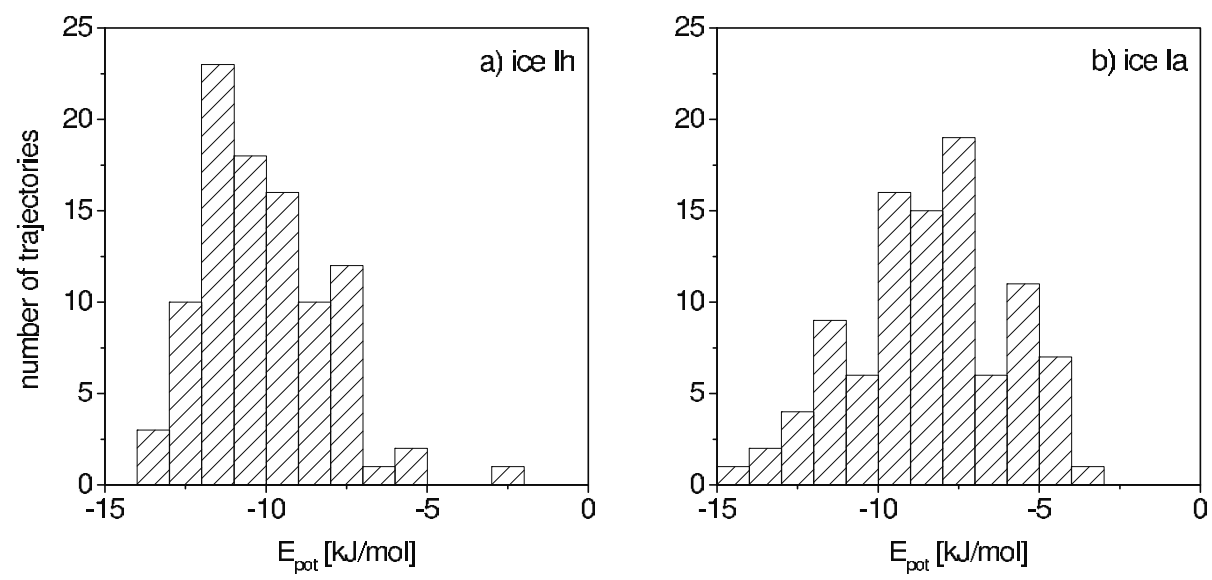

Figure 4. Using histograms, the number of sticking trajectories is shown as a function of the final value of the potential interaction energy of $\mathrm{CO}$ with ice, for $E_{i}=9.6 \mathrm{~kJ} \mathrm{~mol}^{-1}$ and $T_{s}=90$ $\mathrm{K}$, for the case of ice $\mathrm{Ih}(a)$ and ice $\mathrm{I}_{a}(b)$ (Al-Halabi, van Dishoeck, \& Kroes 2004).

From the MD simulations, it is also possible to compute the final potential interaction energy of the collider (CO) with the surface. Distributions of the adsorption energy $\left(E_{a d}\right)$ are shown in Figure 4 for ice $\mathrm{Ih}$ and ice $\mathrm{I}_{a}$ (Al-Halabi, van Dishoeck, \& Kroes 2004). The distribution is broader for ice $\mathrm{I}_{a}$ than for ice Ih. This reflects the greater variety of adsorption sites on ice $\mathrm{I}_{a}$ : the weakest interactions are due to $\mathrm{CO}$ interacting with just a few $\mathrm{H}_{2} \mathrm{O}$ molecules protruding from the irregular surface, and similarly strong interactions can result from $\mathrm{CO}$ interacting with many water molecules, as can happen when $\mathrm{CO}$ moves into a hole in the irregular surface of ice $\mathrm{I}_{a}$ (Fig. 1d). The average adsorption energies extracted from the distributions differ somewhat; i.e., $E_{a d}$ computed for ice $\mathrm{I}_{a}$ was $-8.4 \pm 0.24 \mathrm{~kJ} \mathrm{~mol}^{-1}$, in reasonably good agreement with the result (about $10 \mathrm{~kJ} \mathrm{~mol}^{-1}$ ) of $\mathrm{CO}+$ ice $\mathrm{I}_{a}$ FT-IR experiments (Allouche et al. 1998; Manca et al. 2000; Martin et al. 2002), and $E_{a d}$ computed for ice Ih was $-10.1 \pm 0.20 \mathrm{~kJ} \mathrm{~mol}^{-1}$.

The adsorption geometry of $\mathrm{CO}$ on ice $\mathrm{I}_{a}$ has also been considered (Al-Halabi et al. 2004), in geometry optimisations in which the ice configuration (from an MD simulation) was kept fixed. The calculations suggest that CO interacts most strongly with dangling OH's (Figs. $5 a$ and $5 b$ ), and more weakly with the bonded OH's (Figs. $5 c-5 e$ ). This is in agreement with IR experiments which find the signal attributed to the interaction with dangling $\mathrm{OH}$, which is observed at $2152 \mathrm{~cm}^{-1}$, to be particularly strong in case of other 
a)

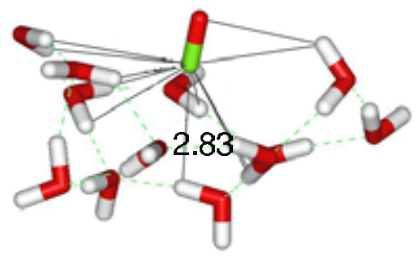

b)

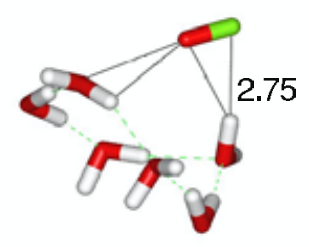

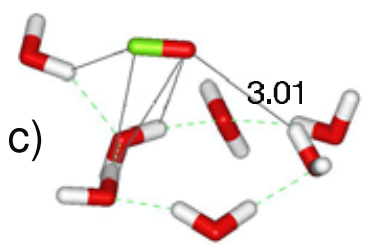

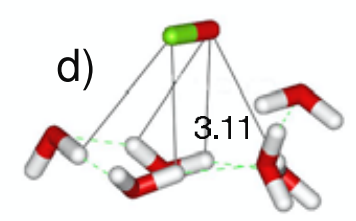

e)

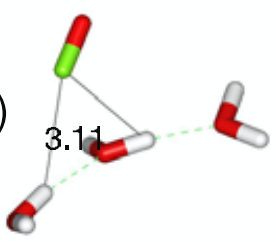

Figure 5. Examples of CO-static ice $\mathrm{I}_{a}$ configurations obtained from geometry optimisations (Al-Halabi et al. 2004). The five examples correspond to five different values of the potential interaction energy of $\mathrm{CO}$ with ice $\mathrm{I}_{a}$, i.e., $(a)-0.155,(b)-0.129,(c)-0.111,(d)-0.090$, and (e) $-0.067 \mathrm{eV}$. Dotted lines illustrate hydrogen bonds between surface water molecules. Solid lines connect $\mathrm{CO}$ with the $\mathrm{H}$-atoms of neighbouring water molecules that are closer to $\mathrm{CO}$ than $3.5 \AA$, the shortest $\mathrm{H}-\mathrm{CO}$ distance being given in $\AA$. Black cylinders represent $\mathrm{O}$ atoms, grey cylinders the $\mathrm{C}$ atom, and light grey spheres the $\mathrm{H}$ atoms. Bonding of $\mathrm{CO}$ to dangling $\mathrm{OH}$ bonds is seen in $(a)$ and $(b)$, while $\mathrm{CO}$ bonds more to "bonded $\mathrm{OH}$ " in $(c),(d)$, and $(e)$.

molecules co-adsorbed at the centres of the hexagonal rings (Devlin 1992; Martin et al. 2002), and absent in case of other molecules co-adsorbed at the dangling OH bonds (Devlin 1992). The absence of the $2152 \mathrm{~cm}^{-1}$ feature in astronomical spectra (Pontoppidan et al. 2003) otherwise clearly showing the presence of $\mathrm{CO}$ can be used as evidence that most of the $\mathrm{CO}$ is not present in intimately mixed $\mathrm{CO}-\mathrm{H}_{2} \mathrm{O}$ ice (Pontoppidan et al. 2003).

$$
\text { 3.3. } H^{+}+\text {Ice }
$$

The $P_{s}$ computed (Cabrera Sanfelix et al. 2005) for $\mathrm{H}^{+}+$ice $\mathrm{Ih}$ is shown as a function of $E_{i}$ in Figure 6 for collisions at normal incidence, at $T_{s}=80 \mathrm{~K}$. The figure shows a surprising result: for low $E_{i}(=0.2 \mathrm{eV}), P_{s}$ is significantly smaller than 1 , and also significantly smaller than $P_{s}$ for $\mathrm{HCl}$, which exceeds 0.9 for these low $E_{i}$ (Al-Halabi et al. 1999; Al-Halabi et al. 2001). This is surprising because the proton's interaction energy with the ice surface (computed to be about $10 \mathrm{eV}$ on average (Cabrera Sanfelix et al. 2005) is much larger (by about a factor 50) than the interaction energy of $\mathrm{HCl}$ with ice (Al-Halabi et al. 1999; Al-Halabi et al. 2001). The explanation for the significant reflection $\left(40 \%\right.$ at $\left.E_{i}=0.05 \mathrm{eV}\right)$ is that in the surface monolayer of ice $\mathrm{Ih} 50 \%$ of the water molecules have an electropositively charged $\mathrm{H}$ atom pointing away from the surface. The impinging proton experiences electrostatic repulsion when impacting close to these molecules, causing it to be reflected at low $E_{i}$. The calculations show that the rule observed for collisions of neutrals with ice (the larger the $E_{a d}$ of the collider on ice, the larger the trapping probability) does not hold when comparing trapping of neutrals with trapping of ions.

The calculations on $\mathrm{H}^{+}+$ice $\mathrm{Ih}$ also yielded another interesting prediction (Cabrera Sanfelix et al. 2005). Figure $7 a$ shows that there is a significant probability (4-16\% for $\mathrm{E}_{i}$ between 0.05 to $3 \mathrm{eV}$ ) that a water molecule desorbs upon impact of the proton (collision induced desorption). The water desorption is not due to "sputtering"; the desorption 


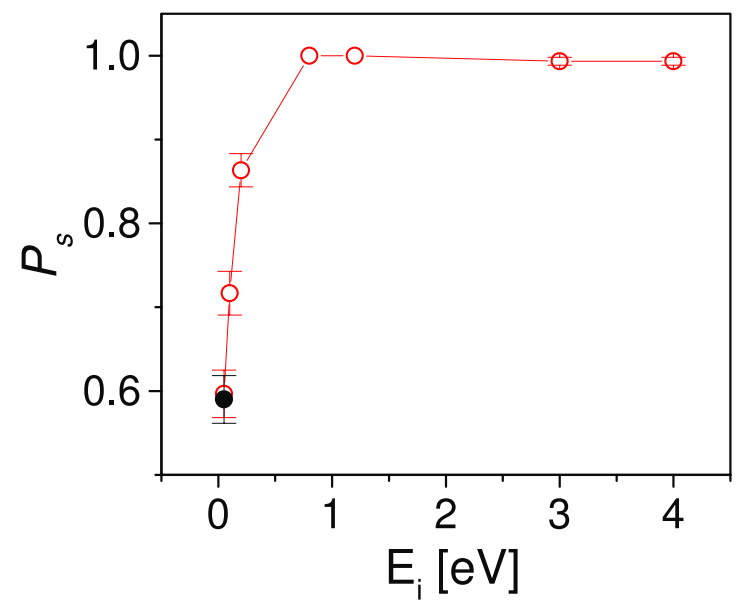

Figure 6. The sticking probability of $\mathrm{H}^{+}$to ice $\mathrm{Ih}$ is shown as a function of $E_{i}$, for normal incidence, at $T_{s}=$ $80 \mathrm{~K}$ (open circles). The solid circle is a result of a control calculation (see Cabrera Sanfelix et al. 2005 for more details).

also occurs at values of $E_{i}$ which are much lower $(0.05 \mathrm{eV})$ than the energy with which surface $\mathrm{H}_{2} \mathrm{O}$ molecules are bound to

the bulk of ice $(>0.3 \mathrm{eV})$. Visualisation of the trajectories show that the water desorption occurs because the stuck proton disrupts the hydrogen bonding network between surface water molecules. The stuck proton can bury itself between the first and the second, or the second and the third bilayer of the ice surface (Fig. 7b). Because the proton's interaction with a neighbouring water molecule $\left(\approx 700 \mathrm{~kJ} \mathrm{~mol}^{-1}\right.$, Table 1$)$ can be much stronger than a hydrogen bond between neighbouring water molecules $(\approx 15-$ $\left.20 \mathrm{~kJ} \mathrm{~mol}^{-1}\right), \mathrm{H}^{+}$can act as a "Coulomb bomb" or "hydrogen bond chain saw", completely disrupting the hydrogen bond network close to it. As a result, a nearby surface water molecule can all of a sudden find itself in a repulsive environment, and desorb.
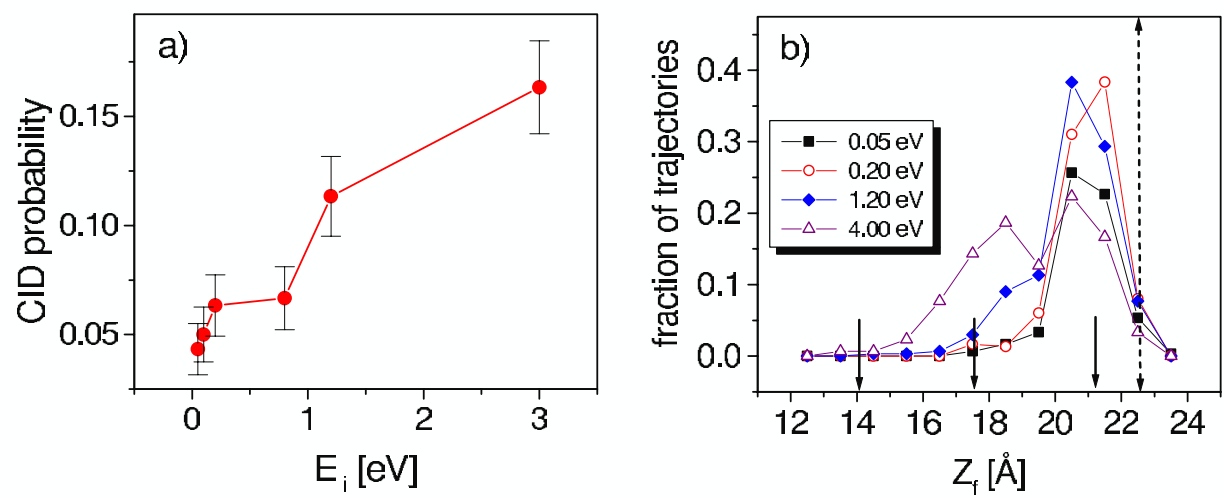

Figure 7. (a) The probability that one $\mathrm{H}_{2} \mathrm{O}$ molecule desorbs upon impact of a proton is shown as a function of $E_{i}$, for $T_{s}=80 \mathrm{~K}$ (Cabrera Sanfelix et al. 2005). (b) Fraction of sticking trajectories plotted for four different $E_{i}$, according to the final value of the co-ordinate for motion normal to the surface, $Z_{f}$ (Cabrera Sanfelix et al. 2005). The positions of the surface bilayers are shown by the arrows at the bottom of the figure. The surface-vacuum interface, indicated by the dashed long double arrow, is located at $22.5 \AA$. 


\subsection{Photodissociation of $\mathrm{H}_{2} \mathrm{O}$ Ice}

In Figure 8 the simulated spectrum of the first absorption band of ice Ih for the old (Andersson et al. 2005) and new (Andersson et al., in prep.) potential models are shown together with the experimental spectrum (Kobayashi 1983) and the calculated first absorption band of gas-phase $\mathrm{H}_{2} \mathrm{O}$. The ice spectra refer to molecules in the third bilayer, which gives a good representation of bulk spectra. The previous model gave a blueshift of $2 \mathrm{eV}$ compared to the gas-phase spectrum, while the new model gives a blueshift of little over $1 \mathrm{eV}$, in good agreement with experiment (Kobayashi 1983). The experimental peak $(8.6 \mathrm{eV})$ and the threshold energy $(7.6 \mathrm{eV})$ are reproduced by the new potential. The calculated peaks of amorphous ice $(8.6 \mathrm{eV})$ and liquid water $(8.2 \mathrm{eV})$ coincide with experimental values (Kobayashi 1983; Heller et al. 1974). These results strongly suggest that the amount of kinetic energy released into the system is also correct.

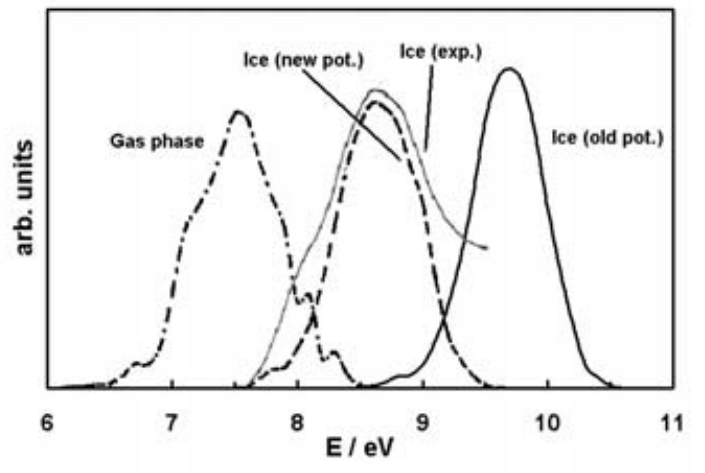

Figure 8. Simulated spectra for the first UV absorption band for ice Ih with the old (Andersson et al. 2005) and new (Andersson et al., in prep.) potential models. Also shown are the calculated spectrum of the first band of gas-phase water UV absorption (Andersson et al. 2005) and the experimental spectrum for ice Ih (Kobayashi 1983).

Figure 9 shows the most important basic outcomes for ice Ih and ice $\mathrm{I}_{a}$ for photodissociation of molecules in the top three bilayers. The case where $\mathrm{H}$ desorbs and $\mathrm{OH}$ becomes trapped is the dominant outcome (60-80\%) in the first bilayer. Already in the second bilayer, trapping of $\mathrm{H}$ and $\mathrm{OH}$ or of recombined water become of roughly equal importance to $\mathrm{H}$ atom desorption. Photodissociation in the third bilayer leads mainly to trapping of $\mathrm{H}$ and $\mathrm{OH}$ or recombined water and only in $10-15 \%$ of the cases to desorption of $\mathrm{H}$. Results for the two types of ice are quite similar with a few noticeable differences. The most prominent difference is that, especially in the first two bilayers, the probability of $\mathrm{H}$ atom desorption is higher for ice $\mathrm{I}_{a}$ than for ice $\mathrm{Ih}$ (1st bilayer: $80 \%$ vs. $65 \%$; 2 nd bilayer: $40 \%$ vs. 25\%). This is explained by the low density of the first bilayer in ice $\mathrm{I}_{a}$, due to its irregular structure. $\mathrm{H}$ atoms moving towards the surface therefore experience fewer collisions in ice $\mathrm{I}_{a}$ than in ice $\mathrm{Ih}$ and have a higher probability of making it into the gas phase.

The $\mathrm{H}$ atoms that become trapped have on average moved about $10 \AA$ from their original position, but atoms travelling over $60 \AA$ have been observed. The trapped $\mathrm{OH}$ radicals on average only move $1-2 \AA$, but in cases where the photodissociation occurs at the surface, the $\mathrm{OH}$ has been found to move much longer distances (up to $85 \AA$ ). This is further than what is found for $\mathrm{H}$ atoms moving over the surface and can be explained by the $\mathrm{OH}$-ice interaction being much stronger than the $\mathrm{H}$-ice interaction. While $\mathrm{H}$ atoms moving at the surface have a high probability of desorbing, the more strongly bound $\mathrm{OH}$ can in most cases not desorb, but it can keep moving parallel to the surface.

Desorption of recombined water molecules is found in $0.2 \%$ of the cases for both types of ice. Indirect water desorption, where one of the molecules not initially photodissociated desorbs, has a probability of $0.2 \%$ for ice $\mathrm{Ih}$ and $0.04 \%$ for ice $\mathrm{I}_{a}$. Note that these 


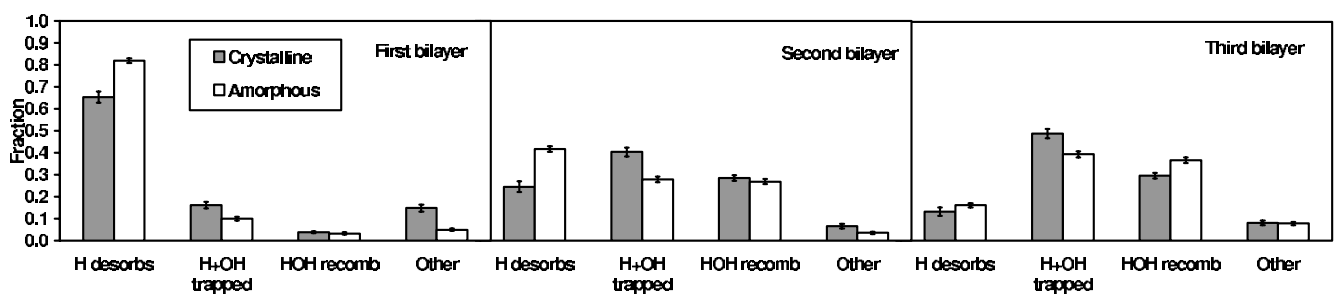

Figure 9. Probabilities (per absorbed UV photon) for the most important basic outcomes of photodissociation of water molecules in the top three bilayers in ice $\mathrm{Ih}$ and $\mathrm{I}_{a}$ (using the new potential model (Andersson et al., in prep.). " $\mathrm{H}$ desorbs" is when $\mathrm{H}$ desorbs and $\mathrm{OH}$ remains trapped, "H+OH trapped" is when $\mathrm{H}$ and $\mathrm{OH}$ become trapped, and "HOH recomb" contains the cases where a recombined water molecule remains trapped. "Other" contains all other outcomes (Andersson et al., in prep.)

probabilities are per absorbed UV photon. The mechanism behind this desorption is through the transfer of momentum of an energetic $\mathrm{H}$ atom to a water molecule, which either desorbs or initiates a further chain of momentum transfer until a molecule at the surface desorbs. The photodesorption of water is found to be quite small, but, in apparent contradiction to our results, Westley et al. (1995) did not detect any desorption of water at low temperatures $(T \leqslant 50 \mathrm{~K})$ in the limit of single-photon absorption. They however used Lyman- $\alpha(10.2 \mathrm{eV})$ photons, which probably leads to a different excited state than the lower excitation energies considered by us.

\section{Conclusions}

We have discussed MD calculations on sticking of atomic hydrogen, $\mathrm{CO}$, and $\mathrm{H}^{+}$to ice $\mathrm{Ih}$ and ice $\mathrm{I}_{a}$; results for $\mathrm{HCl}+$ ice were also briefly mentioned. A general rule that emerged for sticking/trapping of neutrals to ice is that $P_{s}$ increases with the adsorption energy of the collider on ice. This rule does not hold when the sticking of neutrals and ions is compared. For $\mathrm{H}$ and $\mathrm{CO}$, we found that, for normal incidence, the computed sticking probability could be fitted quite well to an exponential function of the collision energy.

For $\mathrm{H}$ and $\mathrm{CO}$, it was possible to compare sticking probabilities computed for interaction with ice Ih and ice $\mathrm{I}_{a}$, in calculations using identical pair potentials for the interaction of the collider with the ice. For CO, only a small difference was found between sticking on ice $\mathrm{Ih}$ and ice $\mathrm{I}_{a}$; for $\mathrm{H}$, the difference was much greater. At present, it is unclear whether this discrepancy is due to differences between the colliders, or to the different ways in which the simulated ice $\mathrm{I}_{a}$ was prepared. The observed discrepancy underlines the need for understanding how the structure of interstellar ice is related to the way it comes about in the ISM, which is a fundamental question of high importance to astrochemistry: water (mostly in ice) is the third abundant molecule in the ISM, and in dense clouds ice surfaces effectively serve as the factory for producing molecular hydrogen, which is the most abundant molecule in the ISM.

Calculations on collisions of neutrals with ice surfaces never show water desorption, even for $E_{i}(2 \mathrm{eV})$ which significantly exceed the binding energies of surface water molecules (a few tenth's of an $\mathrm{eV}$ ). In contrast, calculations here presented show collision induced desorption of water molecules upon impact of protons even at $E_{i}(0.05 \mathrm{eV})$ much smaller than the binding energies of surface water molecules. In the mechanism, the proton, the interaction of which with a neighbouring water molecule can be much stronger 
than a hydrogen bond between neighbouring water molecules, disrupts the hydrogen bonding network, acting like a "Coulomb bomb".

We have also presented results of new calculations on photodissociation of water in ice Ih and ice $\mathrm{I}_{a}$, in the first absorption band. The emphasis was on the dynamical outcome of photoexcitation of a water molecule in the top $10 \AA$ of the ice surface (first 3 bilayers), because photoinitiated chemistry with co-adsorbed molecules, which we are interested in studying next, is most likely to occur at the surface. By adjusting the dipole moment on the excited water molecule, which affects the interaction of that water molecule with the surrounding water molecules, it was possible to reproduce the position and the shape of the first absorption band in the experimental spectrum in a semi-classical calculation.

Subsequent MD calculations show that the most important dynamical outcomes following photoexcitation in the top surface layer are dissociation followed by H-atom desorption (dominant following photoexcitation in the top bilayer) and dissociation followed by trapping of both the $\mathrm{H}$ and $\mathrm{OH}$ photofragments (dominant following photoexcitation in the third bilayer). In contrast, water desorption was found to be a very unlikely outcome $(<0.5 \%)$. The dynamics is usually complete in around $1 \mathrm{ps}$, which is much shorter than the average time-interval between subsequent collisions of UV photons with a typical ice-covered grain in dense clouds (about a week). While there are subtle differences between photodissociation of water in ice $\mathrm{Ih}$ and ice $\mathrm{I}_{a}$, the similarities in the spectrum and the dynamical outcomes are more striking than the differences.

\section{Acknowledgements}

The authors like to thank E.F. van Dishoeck, who contributed importantly to the work here presented. We also thank A. Al-Halabi, who performed the calculations on sticking of $\mathrm{H}$ and $\mathrm{CO}$ to ice, and importantly contributed to the calculations on $\mathrm{H}^{+}+$ ice. We also thank A.W. Kleyn, M.C. van Hemert, H.J. Fraser, P. Cabrera Sanfelix, G.R. Darling, and S. Holloway, whose co-authorship on one or more of the papers discussed here reflects the importance of the contributions they made to the work presented here. We also thank R. van Harrevelt for help with the initial conditions for the calculations on photodissociation. The work of S. Andersson was funded by a NWO/Spinoza grant (for E.F. van Dishoeck), and a CW programme grant. The work was also funded by FOM, and through grants of computer time by the Stichting Nationale computerfaciliteiten $(\mathrm{NCF})$.

\section{References}

Al-Halabi, A., Kleyn, A.W., \& Kroes, G.J. 1999, Chem. Phys. Lett. 307, 505

Al-Halabi, A., Kleyn, A.W., \& Kroes, G.J. 2001, J. Chem. Phys. 115, 482

Al-Halabi, A., Kleyn, A.W., van Dishoeck, E.F., \& Kroes, G.J. 2002, J. Phys. Chem. B 106, 6515

Al-Halabi, A., Kleyn, A.W., van Hemert, M.C., van Dishoeck, E.F., \& Kroes, G.J. 2003, J. Phys. Chem. A 107, 10615

Al-Halabi, A., Fraser, H.J., Kroes, G.J., \& Van Dishoeck, E.F. 2004, A\&A 422, 777

Al-Halabi, A., van Dishoeck, E.F., \& Kroes, G.J. 2004, J. Chem. Phys. 120, 3358

Allamandola, L.J., Sandford, S.A., \& Valero, G.J. 1988, Icarus 76, 225

Allen, M.P. \& Tildesley, D.J. 1987, Computer Simulations of Liquids (Clarendon: Oxford)

Allouche, A., Verlaque, P., \& Pourcin, J. 1998, J. Phys. Chem. B 102, 89

Andersson, S., Kroes, G.J., \& van Dishoeck, E.F. 2005, Chem. Phys. Lett. 408, 415

Andersson, S., Kroes, G.J., \& van Dishoeck, E.F., in preparation

Berendsen, H.J.C., Postma, J.P.M., van Gunsteren, V.F., Dinola, A., \& Haak, J.R. 1984, J. Chem. Phys. 81, 3684 
Bernal, J.D. \& Fowler, R.H. 1933, J. Chem. Phys. 1, 515

Buch, V. \& Zhang, Q. 1991, Ap. J. 379, 647

Cabrera Sanfelix, P., Al-Halabi, A., Darling, G.R., Holloway, S., \& Kroes, G.J. 2005, J. Am. Chem. Soc. 127, 3944

Devlin, J.P. 1992, J. Phys. Chem. 96, 6185

Dobbyn, A.J. \& Knowles, P.J. 1997, Mol. Phys. 91, 1107

Dohnálek, Z., Kimmel, G.A., Ayotte, P., Smith, R.S., \& Kay, B.D. 2003, J. Chem. Phys. 118, 364

Ehrenfreund, P. \& Schutte, W.A. 2000, in Astrochemistry: From Molecular Clouds to Planetary Systems, eds. Y.C. Minh \& E.F. van Dishoeck (ASP: San Francisco), vol. 197, p. 135

Ehrenfreund, P., Fraser, H.J., Blum, J., Cartwright, J.M.E., Garcia-Ruiz, J.M., Hadamcik, E., Levasseur-Regourd, A.C., Price, S., Prodi, F., \& Sarkissian, A. 2003, Plan. Space. Sci. 51, 473

Gerakines, P.A., Moore, M.H., \& Hudson, R.L. 2000, A\&A 357, 793

Graham, A.P., Menzel, A., \& Toennies, J.P. 1999, J. Chem. Phys. 111, 1169

Hagen, W., Tielens, A.G.G.M., \& Greenberg, J.M. 1981, Chem. Phys. 56, 257

Heller, J.M., Jr., Hamm, R.N., Birkhoff, R.D., \& Painter, L.R. 1974, J. Chem. Phys. 60, 3483

Herbst, E. 1995, Annu. Rev. Phys. Chem. 46, 27

Hollenbach, D. \& Salpeter, E.E. 1971, Ap. J. 163, 155

Jorgensen, W.L., Chandrasekhar, J., Madura, J.D., Impey, R.W., \& Klein, M.L. 1983, J. Chem. Phys. 79, 926

Karim, O.A. \& Haymet, A.D.J. 1988, J. Chem. Phys. 89, 6889

Klein, S., Kochanski, E., \& Strich, A. 1996, Theor. Chim. Acta 94, 75

Kobayashi, K. 1983, J. Phys. Chem. 87, 4317

Kobayashi, K., Kasamatsu, T., Kaneko, T., Koike, J., Oshima, T., Saito, T., Yamamoto, T., \& Yanagawa, H. 1995, Adv. Space Res. 16, 21

Kozack, R.E. \& Jordan, P.C. 1992, J. Chem. Phys. 96, 3131

Kroes, G.J. 1992, Surf. Sci 275, 365

Maldoni, M.M., Robinson, G., Smith, R.G., Duley, W.W., \& Scott, A. 1999, MNRAS 309, 325

Manca, C, Roubin, P., \& Martin, C. 2000, Chem. Phys. Lett. 330, 21

Manicó, G., Raguni, G., Pironello, V., Roser, J.E., \& Vidali, G. 2001, Ap. J. 548, L253

Martin, C, Manca, C., \& Roubin, P. 2002, Surf. Sci. 502-503, 280

Masuda, K., Takahashi, J., \& Mukai, T. 1998, A\&3A 330, 773

Mukai, T. \& Schwehm, G. 1981, A, \& A 95, 373

Petrenko, V.F. \& Whitworth, R.W. 1999, Physics of Ice (University Press: Oxford)

Pontoppidan, K.M., Fraser, H.J., Dartois, E., Thi, W.F., van Dishoeck, E.F., Boogert, A.C.A., d'Hendecourt, L., Tielens, A.G.G.M., \& Bisschop, S.E. 2003, A\&JA 408, 981

Porter, R.N. \& Raff, L.M. 1976, in Dynamics of Molecular Collisions, Part B, ed. W.H. Miller (Plenum: New York), p. 1

Schinke, R. 1993, Photodissociation Dynamics (Cambridge University Press: Cambridge)

Takahashi, J., private communication

Tanaka, M., Nagata, T., Sato, S., Yamamoto, T. 1994, Ap. J. 430, 779

van Dishoeck, E.F. 1998, in The Molecular Astrophysics of Stars and Galaxies, eds. T.W. Hartquist \& D.A. Williams (Clarendon Press: Oxford), p. 53

van Harrevelt, R. \& van Hemert, M.C. 2000, J. Chem. Phys. 112, 5777

van Harrevelt, R., van Hemert, M.C., \& Schatz, G.C. 2001, J. Phys. Chem. A 105, 11480

Watanabe, N. \& Kouchi, A. 2002, Ap. J. 567, 651

Westley, M.S., Baragiola, R.A., Johnson, R.E., \& Baratta, G.A. 1995, Nature 373, 405

Yaron, D., Peterson, K.I., Zolandz, D., Klemperer, W., Lovas, F.J., \& Suenram, R.D. 1990, J. Chem. Phys. 92, 7095

Zhang, Q., Sabelli, N., \& Buch, V. 1991, J. Chem. Phys. 95, 1080

\section{Discussion}

HERBST: What do you suspect happens when high energy ions strike crystalline and amorphous ice? 
Kroes: Brown et al. have studied scattering of high energy protons (0.5 MeV and 1.5 MeV, respectively) from amorphous ice (1978, Phys. Rev. Lett. 40, 1027). Their most important finding was that the sputtering yield (number of water molecules sputtered per incident ion) was 0.4 for a collision energy of $0.5 \mathrm{MeV}$, and 0.2 for $1.5 \mathrm{MeV}$. Extrapolating to the most usual energies of cosmic rays (approximately $100 \mathrm{MeV}$ ), one would think that at such high incidence energies no sputtering would be observed at all. I know of no experiments that have been done at these high energies. A recent review discussing sputtering of ice by highly energetic positive ions is that of Baragiola et al. (2003, Nucl. Instruym. Methods Phys. Res. B 209, 294), who mention that the experiments of Brown et al. (at 0.5 and $1.5 \mathrm{MeV}$ ) suggest that sputtering is an important phenomenon on icy satellites and grains subject to irradiation by energetic ions from solar flares and by magnetospheric ions. 